\title{
Self-organized electromagnetic field structures in laser-produced counter-streaming plasmas
}

\author{
N. L. Kugland ${ }^{1 \star}$, D. D. Ryutov' , P-Y. Chang ${ }^{2}$, R. P. Drake ${ }^{3}$, G. Fiksel ${ }^{2}$, D. H. Froula ${ }^{2}$, S. H. Glenzer ${ }^{1}$, \\ G. Gregori ${ }^{4}$, M. Grosskopf ${ }^{3}$, M. Koenig ${ }^{5}$, Y. Kuramitsu ${ }^{6}$, C. Kuranz ${ }^{3}$, M. C. Levy ${ }^{1,7}$, E. Liang ${ }^{7}$, \\ J. Meinecke ${ }^{4}$, F. Miniati ${ }^{8}$, T. Morita ${ }^{6}$, A. Pelka ${ }^{5}$, C. Plechaty ${ }^{1}$, R. Presura9 ${ }^{9}$, A. Ravasio ${ }^{5}$, \\ B. A. Remington ${ }^{1}$, B. Reville ${ }^{4}$, J. S. Ross ${ }^{1}$, Y. Sakawa ${ }^{6}$, A. Spitkovsky ${ }^{10}$, H. Takabe ${ }^{6}$ and H-S. Park ${ }^{1}$
}

Self-organization ${ }^{1,2}$ occurs in plasmas when energy progressively transfers from smaller to larger scales in an inverse cascade $^{3}$. Global structures that emerge from turbulent plasmas can be found in the laboratory ${ }^{4}$ and in astrophysical settings; for example, the cosmic magnetic field ${ }^{5,6}$, collisionless shocks in supernova remnants ${ }^{7}$ and the internal structures of newly formed stars known as Herbig-Haro objects ${ }^{8}$. Here we show that large, stable electromagnetic field structures can also arise within counter-streaming supersonic plasmas in the laboratory. These surprising structures, formed by a yet unexplained mechanism, are predominantly oriented transverse to the primary flow direction, extend for much larger distances than the intrinsic plasma spatial scales and persist for much longer than the plasma kinetic timescales. Our results challenge existing models of counter-streaming plasmas and can be used to better understand large-scale and long-time plasma self-organization.

Our experiments were performed at the OMEGA EP laser facility, where two kilojoule-class lasers irradiated two polyethylene $\left(\mathrm{CH}_{2}\right)$ plastic discs that faced each other at a distance of $8 \mathrm{~mm}$, creating a system of high-velocity laser-ablated counter-streaming plasma flows. The experimental details are described in Fig. 1 and in the Methods. At early times, up to at least $8 \mathrm{~ns}$, intra-jet ion collisions are known to be strong (owing to relatively low-particle thermal velocities) but inter-jet ion collisions are rare (owing to relatively high flow velocities), permitting the evolution of both hydrodynamic and collisionless plasma instabilities ${ }^{9,10}$ (Table 1). We visualized the electric and magnetic field structures in the counter-streaming plasmas with short-pulse laser-generated proton beam imaging ${ }^{11,12}$, taken from two orthogonal views to evaluate the possible azimuthal symmetry of the field structures. After roughly $3 \mathrm{~ns}$, caustics (large-intensity variations ${ }^{13}$ ) in the proton images indicate the formation of strong field zones within the plasma, probably due to sharp structures with strong gradients, as reported elsewhere ${ }^{14}$. By 4 ns, the features have changed markedly into two large swaths of straight transverse caustics that extend for up to $5 \mathrm{~mm}$. This extent is large compared with the fundamental scale lengths of the plasma (Table 1$)$ such as the Debye length $(50,000$ times larger) and the ion inertial length (nearly 100 times larger), indicating a high degree of self-organization. This organization

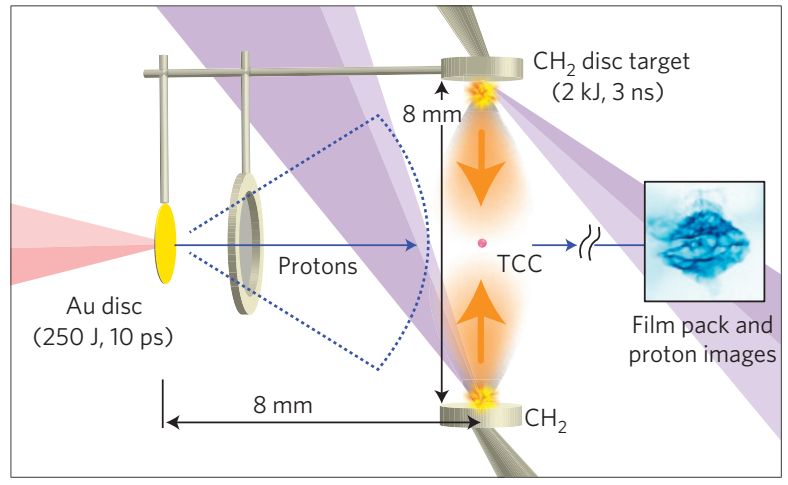

Figure 1 | Experimental set-up at the OMEGA EP laser showing the targets, laser beams and diagnostic configuration. Two long-pulse lasers (purple) created counter-streaming plasmas from $\mathrm{CH}_{2}$ disc targets. We visualized the fields in these plasmas with short-pulse (red)

laser-generated protons from two orthogonal views (only one line of sight is shown). The location of the target chamber centre (TCC) is marked with a magenta dot in the middle. Typical radiochromic film data are shown in the inset image.

proceeds up to a spatial scale that is comparable to the size of the system (that is, the disc separation). These caustics remain in place from 4 to $7 \mathrm{~ns}$, the remaining duration of the experimental window, indicating a high degree of stability. This 3 ns lifetime is long compared with fundamental plasma timescales: 75,000 times longer than the electron plasma period, nearly 3,000 times longer than the ion plasma period, and $>30$ times longer than the flow crossing time across the $\approx 100$ - $\mu$ m-wide structure thickness. Scaled laboratory astrophysics experiments have demonstrated significant potential to enhance our understanding of the generation and evolution of fields in galactic- and extra-galactic environments ${ }^{15-18}$, and may be able to help address several major outstanding questions in astrophysics. For example, large-scale magnetic fields have been observed in young stellar objects ${ }^{19}$ and are believed to drive the formation of their outflows ${ }^{20}$ and increase the order of the flow ${ }^{21}$. However, the precise role of electromagnetic fields in shaping the large-scale structure of the associated jets and

\footnotetext{
${ }^{1}$ Lawrence Livermore National Laboratory, Livermore, California 94550, USA, ${ }^{2}$ Laboratory for Laser Energetics, University of Rochester, Rochester, New York 14636, USA, ${ }^{3}$ Department of Atmospheric, Oceanic, and Space Sciences, University of Michigan, Ann Arbor, Michigan 48109, USA, ${ }^{4}$ Department of Physics, University of Oxford, Parks Road, Oxford OX1 3PU, UK, ${ }^{5}$ Laboratoire pour I'Utilisation des Lasers Intenses (LULI), École Polytechnique-Univ, Paris VI, 91128 Palaiseau, France, ${ }^{6}$ Institute of Laser Energetics, Osaka University, Osaka 565-0871, Japan, ${ }^{7}$ Rice University, Houston, Texas 77251, USA, ${ }^{8}$ Physics Department, Wolfgang-Pauli-Strasse 27, ETH-Zürich, CH-8093 Zürich, Switzerland, ${ }^{9}$ University of Nevada, Reno, 1664 North Virginia Street, Reno, Nevada 89557, USA, ${ }^{10}$ Princeton University, Princeton, New Jersey 08544, USA. *e-mail: kugland1@llnl.gov.
} 
Table 1 | Typical plasma parameters for our counter-streaming $\mathrm{CH}_{2}$ plasmas at 4 ns at the TCC.

\begin{tabular}{|c|c|c|c|c|c|c|c|}
\hline $\begin{array}{l}\text { Electron } \\
\text { density } \\
n_{\mathrm{e}}\left(\mathrm{cm}^{-3}\right)\end{array}$ & $\begin{array}{l}\text { Electron } \\
\text { temperature } \\
T_{\mathrm{e}}(\mathrm{eV})\end{array}$ & $\begin{array}{l}\text { Ion tem- } \\
\text { perature } \\
T_{\mathrm{i}}(\mathrm{eV})\end{array}$ & $\begin{array}{l}\text { Flow velocity } \\
v_{\text {flow }}\end{array}$ & $\begin{array}{l}\text { Inter-jet } \\
\text { collisional mean } \\
\text { free path, } \lambda_{\mathrm{mfp}}\end{array}$ & $\begin{array}{l}\text { Debye length, } \\
\lambda_{\text {Debye }}\end{array}$ & $\begin{array}{l}\mathrm{CH}_{2} \text { ion plasma } \\
\text { period, } \\
\tau_{\mathrm{pi}}=2 \pi / \omega_{\mathrm{pi}}\end{array}$ & $\begin{array}{l}\mathrm{CH}_{2} \text { ion } \\
\text { inertial } \\
\text { length, } c / \omega_{\mathrm{pi}}\end{array}$ \\
\hline $8 \times 10^{18}$ & 1,000 & 1,500 & $\begin{array}{l}10^{8} \mathrm{~cm} \mathrm{~s}^{-1} \\
\left(1 \mathrm{~mm} \mathrm{~ns}^{-1}\right) \\
\text { Mach 3-5 }\end{array}$ & $\begin{array}{l}250 \mathrm{~mm}(\mathrm{HH}), \\
56 \mathrm{~mm}(\mathrm{CC})\end{array}$ & $0.1 \mu \mathrm{m}$ & $1.1 \mathrm{ps}$ & $51 \mu \mathrm{m}$ \\
\hline
\end{tabular}

We measured these with Thomson scattering at the OMEGA laser under similar target and laser conditions ${ }^{9}$. The mean free path listed is for ion-ion collisions between flows (inter-jet); HH and CC refer to hydrogen-hydrogen and carbon-carbon collisions, respectively. The fully ionized multispecies ion plasma frequency is calculated as $\omega_{\mathrm{pi}}^{2}=\omega_{\mathrm{pi}[\mathrm{C}]}^{2}+2 \omega_{\mathrm{pi}[\mathrm{H}]}^{2}$, where the factor of 2 comes from the presence of two hydrogen atoms per carbon atom.
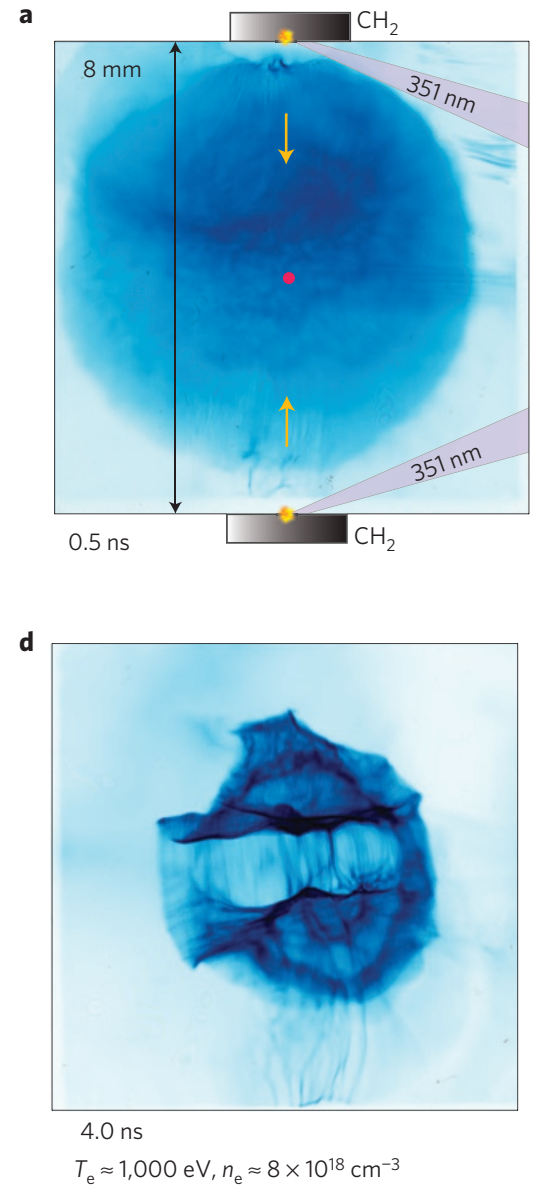

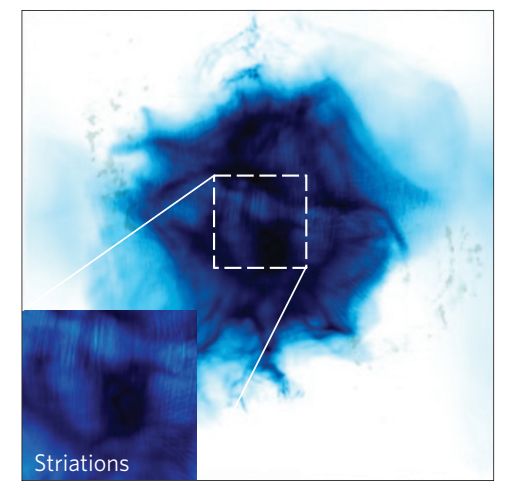

$2.2 \mathrm{~ns}$

$T_{\mathrm{e}} \approx 500 \mathrm{eV}, \mathrm{n}_{\mathrm{e}} \approx 3 \times 10^{18} \mathrm{~cm}^{-3}$

e

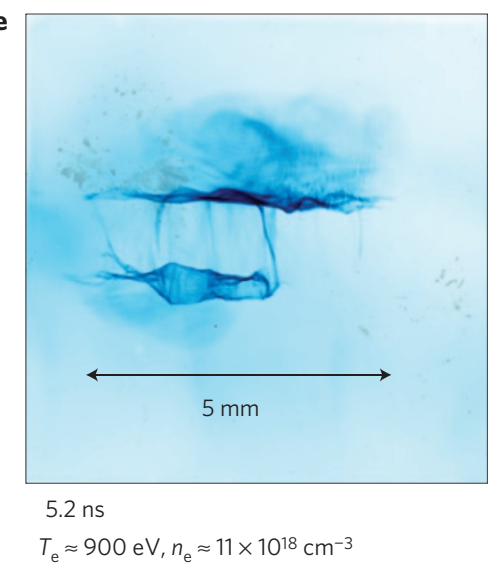

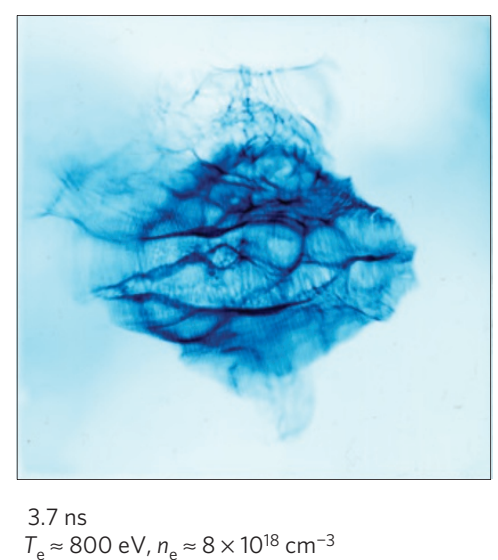

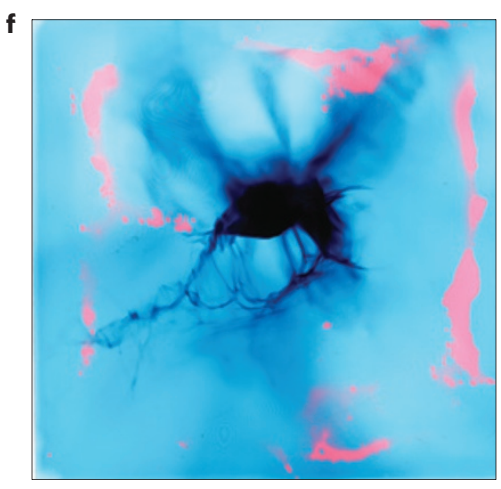

$7.0 \mathrm{~ns}$

Figure $\mathbf{2}$ | Side-view time sequence of proton images showing the evolution of self-organized electromagnetic field structures. Dimensions are given in estimated object plane sizes. a, At early time the plasmas are still close to the targets, and the blotchiness in the centre of the image is from weak modulations in the proton beam itself. The orange arrows show the direction of counter-streaming flow. The magenta dot marks the TCC. $\mathbf{b}$, Turbulence and striations develop. c, Caustics appear. d-f, Large-scale caustics appear (d) and persist (e) out to $7 \mathrm{~ns}(\mathbf{f})$. The times indicated are when the protons in the centre of each image reach the TCC, that is, the sum of the short-pulse laser delay and the proton time of flight. a-e have proton energy $W=8.8 \mathrm{MeV}$; f has $W=4.7 \mathrm{MeV}$. Image contrast has been individually adjusted. We measured the electron temperatures $T_{e}$ and densities $n_{e}$ at the TCC with Thomson scattering (see Table 1).

Herbig-Haro objects is unknown ${ }^{8}$. Furthermore, laser-produced plasmas may be capable of collisionless shock formation ${ }^{10,22,23}$. The role of astrophysical magnetic field generation at shocks may affect protogalactic structure formation ${ }^{18,24}$, a possibility that seems more likely because the existence of coherent fields in galaxies has recently been observed ${ }^{25}$. In all of these astrophysical objects, as in our experiment, large-scale field structures are clearly identified. As different plasma instabilities probably dominate each of these systems, our work should be considered as a test bed for studying the general physics of self-organization in plasmas.
The electromagnetic fields in the counter-streaming $\mathrm{CH}_{2}$ plasmas are visualized in the proton image time sequence of Fig. 2. This side-view sequence, which was obtained over six separate shots in which the proton beam delay was steadily increased, shows at a glance how the fields evolve. We note that for this millimetre-scale plasma with a density near $10^{19} \mathrm{~cm}^{-3}$ (areal density near $10^{18} \mathrm{~cm}^{-2}$ ), the proton imagery is created purely by electric and magnetic fields, with only negligible collisional scattering of the proton beam.

At early times of $0.5 \mathrm{~ns}$ (Fig. 2a) small plasmas expand in isolation away from the $\mathrm{CH}_{2}$ targets. By $2.2 \mathrm{~ns}$ (Fig. 2b) the 

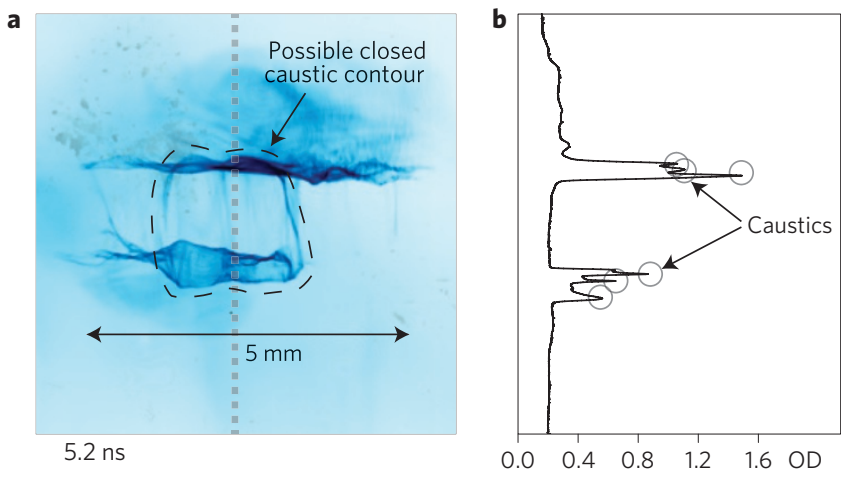

Figure 3 | Caustic detail. a, A detail of the proton image from Fig. 2d, with the dashed line indicating a nearly closed contour suggestive of a cellular field structure. $\mathbf{b}$, A line profile (in units of optical density, OD) taken along the dotted line in $\mathbf{a}$, from a separate scan of the film with a photometric densitometer. The sharp features, circled, are caustics made by self-organized field structures.

dominant image features are general turbulence as well as fine striations $(10 \mu \mathrm{m}$ scale) that are oriented along the counterstreaming (vertical) axis. By $3.7 \mathrm{~ns}$ (Fig. 2c) the bulk populations of the two plasma flows have almost met, and the turbulent features have sharpened into strong caustics ${ }^{14}$, many circular with a spatial scale of roughly $0.5 \mathrm{~mm}$, along with a hint of longerrange organization along the horizontal direction. The sharp, clear features in these proton images imply a dominant curtain structure of fields, or possibly the presence of several (but not too many) volumetric field structures within the field of view. The presence of volumetric structures on a small scale would cause only blurring ${ }^{14}$.

At $4.0 \mathrm{~ns}$ (Fig. 2d) the features have changed markedly from general turbulence to a strongly self-organized regime with two large swaths of horizontal caustics separated by roughly $1.5 \mathrm{~mm}$ in the object plane. These features seem to be up to $5 \mathrm{~mm}$ long and stand in place from approximately $4-7$ ns (Fig. $2 d-f$ ). This long lifetime implies that the plasma field structures are in a stable steady state. As detailed in Fig. 3a, there are nearly closed caustic contours connecting the two horizontal features, suggesting a cellular field structure. The horizontal swaths themselves consist of multiple caustics clustered together, as shown in Fig. 3b. The field structures that create this feature are roughly axisymmetric about the vertical axis, because images from the orthogonal proton beam (not shown) look similar. The features have a generally bubbly appearance that might be caused by hydrodynamic turbulence or other instability mechanisms.

The positions of the caustics in Fig. $2 \mathrm{~d}$ remain the same for images with proton energies from 7 to $15 \mathrm{MeV}$ (see Supplementary Fig. S1), indicating that: the structures must change over a time that is longer than the proton beam temporal spread, roughly $100 \mathrm{ps}$; the features are created by sharp field structures. Proton deflection angles $\alpha$ have a well-known scaling with the proton energy $W$, namely $\alpha \propto W^{-1}$ for electric deflections and $\alpha \propto W^{-1 / 2}$ for magnetic deflections ${ }^{14}$. However, proton caustics caused by sharp spatial structures possess a special stability in the high-magnification regime: the gross position of the caustic is insensitive to $W$, and only the positions of the individual caustic branches (which are too finely spaced to individually resolve) are sensitive to $W$ (ref. 14).

To better understand the origin of our observed field structures, let us consider the ingredients that are present. We know that the jets interpenetrate and that collisional stagnation of the two flows cannot occur in the time window during which we have proton images, although collisions do occur ${ }^{9}$. Conditions are appropriate to support the growth of electrostatic and electromagnetic plasma instabilities ${ }^{22,23,26,27}$. Strong and rapid electron and ion heating occurs around 2.5-3.5 ns, raising both temperatures by more than an order of magnitude ${ }^{9}$. This heating impacts both the visibility of electrostatic structures for proton imaging (electrostatic proton deflection $\sim$ electric field $\sim T_{\mathrm{e}}$ ) and the plasma dynamics (sound speed $\sim$ $T_{\mathrm{e}}^{1 / 2}$ ). Indeed, the high temperature might explain the dominance of the caustic features, which have not been seen with such clarity in previous proton imaging experiments (see references in ref. 14). Intra-jet shocks are expected to be present and might contribute to the heating and formation of large-scale structures ${ }^{28}$. Electrostatic structures with potentials of the order of the electron temperature seem the most plausible, given that relatively high magnetic fields (tens of teslas) would be required to create caustics in these proton images ${ }^{14}$. Although large magnetic fields are produced at the laser spot $^{29}$, they are subsequently advected and volumetrically diluted during the plasma expansion ${ }^{23}$. Consequently, in the volume near the target chamber centre (Fig. 1) the counter-streaming plasmas are essentially unmagnetized, an interesting regime that is not well explored. The main caustic features are summarized in Table 2.

Although the origin of the fields that create the horizontal swaths of caustics is still unknown, we can nevertheless present a phenomenological description. These swaths of caustics could be from planar field structures, or the rims of conical or cylindrical discontinuities, seen side-on. A central blob of field might exist, possibly with a cellular structure, in which the upper and lower edges are the most visible. Our detailed analysis of proton imaging ${ }^{14}$ suggests that two widely separated layers of fields are required to create two widely separated swaths of caustics; for our experimental regime, a single field layer produces only a pair of very finely spaced caustics that appear as one on the detector. However, exact determination of the volume occupied by the fields (including the corresponding object plane sizes) will require further experimental work: one might vary the proton source distance, film distance and viewing angle.

Our work shows the emergence of large, stable, self-organized fields in counter-streaming plasmas. These structures persist for thousands of ion- and tens of thousands of electron-kinetic timescales. Although it is not entirely clear how these structures form, evidence of their existence is clearly seen in the experimental data. This highly nonlinear regime seems to be beyond the reach of self-consistent simulations: at the present time, there are no threedimensional simulations that can correctly resolve the nonlinear

Table 2 | Proton image features, properties and possible origins.

\begin{tabular}{llll} 
Caustic feature & Spatial scale (object plane) & Most visible & Possible origins \\
\hline $\begin{array}{lll}\text { Striations } \\
\text { Turbulent circular caustics }\end{array}$ & $\begin{array}{l}10 \mu \mathrm{m} \\
0.5 \mathrm{~mm}\end{array}$ & $\begin{array}{l}2 \mathrm{~ns} \\
2-3 \mathrm{~ns}\end{array}$ & $\begin{array}{l}\text { Shocks in a sheared flow } \\
\text { Hydrodynamic instabilities (for example, laser ablative or } \\
\text { Rayleigh-Taylor) }\end{array}$ \\
$\begin{array}{lll}\text { Dual swaths of horizontal caustics } \\
1 \mathrm{~mm}\end{array}$ & $4-7 \mathrm{~ns}$ & $\begin{array}{l}\text { Requires a highly self-organizing inverse cascade mechanism. } \\
\text { The outcome field structure could be dual planar, } \\
\text { cylindrical/conical or a single wavy blob }\end{array}$ \\
Nearly closed contours & $1 \mathrm{~mm}$ & $4-7 \mathrm{~ns}$ & $\begin{array}{l}\text { Cellular field structures } \\
\text { wand }\end{array}$
\end{tabular}


plasma instabilities, ion kinetics and structure formation to obtain agreement with the experimental results shown here.

\section{Methods}

The symmetric experimental configuration used two long-pulse (2,200 J, $3 \mathrm{~ns})$ $351 \mathrm{~nm}$ laser beams that focused with an intensity of approximately $3 \times 10^{15} \mathrm{~W} \mathrm{~cm}^{-2}$ (elliptical spot with major and minor diameters of roughly $340 \mu \mathrm{m} \times 100 \mu \mathrm{m}$, respectively) onto two 2 -mm-diameter $\times 0.5$ - $\mathrm{mm}$-thick $\mathrm{CH}_{2}$ disc targets to create the counter-streaming plasmas. We performed proton imaging with two short-pulse (250 J, $10 \mathrm{ps)} 1,053 \mathrm{~nm}$ laser beams focused to $2 \times 10^{18} \mathrm{~W} \mathrm{~cm}^{-2}$ onto two 2 -mm-diameter $\times 50-\mu \mathrm{m}$-thick Au disc targets (only one is shown). These proton sources were located relatively far from the TCC at a distance of $8 \mathrm{~mm}$ to use the entire $\approx f / 1$ proton beam to see from one $\mathrm{CH}_{2}$ target to the other $\mathrm{CH}_{2}$ target. (We consider the object plane to be the plane parallel to the proton source foil surface that passes through the TCC.) The proton imaging magnification $M \approx 7.25$. Two $\mathrm{Al}$ washers, of $2.7 \mathrm{~mm}$ inside diameter, covered with 3 - $\mu \mathrm{m}$-thick $\mathrm{Al}$ foil protected the surface of the Au foil from perturbation by long-pulse $\mathrm{CH}_{2}$ plasma $^{30}$. To avoid interference, we delayed the two proton beams by $0.5 \mathrm{~ns}$ with respect to each other by staggering the short-pulse laser timing. We collected the protons on radiochromic film layered with $\mathrm{Al}$ foil filters to obtain relatively narrowband images with protons from 5 to $15 \mathrm{MeV}$.

\section{Received 30 May 2012; accepted 28 August 2012; published online 30 September 2012}

\section{References}

1. Ball, P. The Self-Made Tapestry: Pattern Formation in Nature (Oxford Univ. Press, 1999).

2. Cross, M. C. \& Hohenberg, P. C. Pattern formation outside of equilibrium. Rev. Mod. Phys. 65, 851-1112 (1993).

3. Diamond, P. H., Itoh, S-I. \& Itoh, K. in Modern Plasma Physics (Physical Kinetics of Turbulent Plasmas, Vol. 1, Cambridge Univ. Press, 2010).

4. Yamada, T. et al. Anatomy of plasma turbulence. Nature Phys. 4, 721-725 (2008).

5. Zweibel, E. G. \& Heiles, C. Magnetic fields in galaxies and beyond. Nature 385, 131-136 (1997).

6. Ryu, D., Kang, H., Cho, J. \& Das, S. Turbulence and magnetic fields in the large-scale structure of the Universe. Science 320, 909-912 (2008).

7. Spicer, D. S., Maran, S. P. \& Clark, R. W. A model of the pre-Sedov expansion phase of supernova remnant-ambient plasma coupling and X-ray emission from SN 1987A. Astrophys. J. 356, 549-571 (1990).

8. Reipurth, B. \& Bally, J. Herbig-Haro flows: Probes of early stellar evolution. Annu. Rev. Astronom. Astrophys. 39, 403-455 (2001).

9. Ross, J. S. et al. Characterizing counter-streaming interpenetrating plasmas relevant to astrophysical collisionless shocks. Phys. Plasmas 19, 056501 (2012).

10. Park, H-S. et al. Studying astrophysical collisionless shocks with counterstreaming plasmas from high power lasers. High Energy Density Phys. 8, 38-45 (2012).

11. Hatchett, S. P. et al. Electron, photon, and ion beams from the relativistic interaction of petawatt laser pulses with solid targets. Phys. Plasmas 7, 2076-2082 (2000).

12. Snavely, R. A. et al. Intense high-energy proton beams from petawatt-laser irradiation of solids. Phys. Rev. Lett. 85, 2945-2948 (2000).

13. Nye, J. F. Natural Focusing and Fine Structure of Light: Caustics and Wave Dislocations (Institute of Physics Publishing, 1999).

14. Kugland, N. L., Ryutov, D. D., Plechaty, C., Ross, J. S. \& Park, H-S. Relation between electric and magnetic field structures and their proton-beam images. Rev. Sci. Instrum. 83, 101301 (2012).

15. Remington, B. A., Arnett, D., Drake, R. P. \& Takabe, H. Modeling astrophysical phenomena in the laboratory with intense lasers. Science 284, 1488-1493 (1999).
16. Ryutov, D. et al. Similarity criteria for the laboratory simulation of supernova hydrodynamics. Astrophys. J. 518, 821-832 (1999).

17. Remington, B. A., Drake, R. P. \& Ryutov, D. D. Experimental astrophysics with high power lasers and Z pinches. Rev. Mod. Phys. 78, 755-807 (2006).

18. Gregori, G. et al. Generation of scaled protogalactic seed magnetic fields in laser-produced shock waves. Nature 481, 480-483 (2012).

19. Ray, T. P. et al. Large-scale magnetic fields in the outflow from the young stellar object T Tauri S. Nature 385, 415-417 (1997).

20. Hartigan, P., Frank, A., Varniere, P. \& Blackman, E. G. Magnetic fields in stellar jets. Astrophys. J. 661, 910-918 (2007).

21. Gardiner, T. A., Frank, A., Jones, T. W. \& Ryu, D. Influence of magnetic fields on pulsed, radiative jets. Astrophys. J. 530, 834-850 (2000).

22. Takabe, H. et al. High-Mach number collisionless shock and photo-ionized non-LTE plasma for laboratory astrophysics with intense lasers. Plasma Phys. Control. Fusion 50, 124057 (2008).

23. Drake, R. P. \& Gregori, G. Design considerations for unmagnetized collisionless-shock measurements in homologous flows. Astrophys. J. 749, 171-185 (2012).

24. Kulsrud, R. M., Cen, R., Ostriker, J. P. \& Ryu, D. The protogalactic origin for cosmic magnetic fields. Astrophys. J. 480, 481-491 (1997).

25. Bernet, M. L., Miniati, F., Lilly, S. J., Kronberg, P. P. \& Dessauges-Zavadsky, M. Strong magnetic fields in normal galaxies at high redshift. Nature 454, 302-304 (2008)

26. Kato, T. N. \& Takabe, H. Nonrelativistic collisionless shocks in unmagnetized electron-ion plasmas. Astrophys. J. 681, L93-L96 (2008).

27. Kato, T. N. \& Takabe, H. Electrostatic and electromagnetic instabilities associated with electrostatic shocks: Two-dimensional particle-in-cell simulation. Phys. Plasmas 17, 032114 (2010).

28. Ryutov, D. D. et al. Intra-jet shocks in two counter-streaming, weakly collisional plasma jets. Phys. Plasmas 19, 074501 (2012).

29. Stamper, J. A. et al. Spontaneous magnetic fields in laser-produced plasmas. Phys. Rev. Lett. 26, 1012-1015 (1971).

30. Zylstra, A. B. et al. Using high-intensity laser-generated energetic protons to radiograph directly driven implosions. Rev. Sci. Instrum. 83, 013511 (2012).

\section{Acknowledgements}

We thank the staff of the OMEGA EP laser facility for their experimental support. This work was performed under the auspices of the US Department of Energy by the Lawrence Livermore National Laboratory, under Contract No. DE-AC52-07NA27344. Further support was provided by LLNL LDRD grant No. 11-ERD-054 and the International Collaboration for High Energy Density Science (ICHEDS), supported by the Core-to-Core Program of the Japan Society for the Promotion of Science. The research leading to these results received funding from the European Research Council under the European Community's Seventh Framework Programme (FP7/2007-2013), ERC grant agreement nos 256973 and 247039.

\section{Author contributions}

N.L.K. and H-S.P. designed and prepared the experiment. The OMEGA EP experiments were carried out by N.L.K., H-S.P., G.G., M.K., Y.K., J.M., T.M., A.P., C.P., J.S.R. and Y.S. The paper was written by N.L.K., D.D.R. and G.G. The data were analysed by N.L.K. and D.D.R. Further experimental and theoretical support was provided by P-Y.C., R.P.D., G.F., D.H.F., S.H.G., M.G., C.K., M.C.L., E.L., F.M., R.P., A.R, B.A.R., B.R., A.S. and H.T.

\section{Additional information}

Supplementary information is available in the online version of the paper. Reprints and permissions information is available online at www.nature.com/reprints. Correspondence and requests for materials should be addressed to N.L.K.

\section{Competing financial interests}

The authors declare no competing financial interests. 